of Sachs and colleagues provides fresh insight into how integrin-dependent tight binding of podocytes to the GBM regulates glomerular disease.

\section{Acknowledgments}

A. Pozzi and R. Zent are supported by an O'Brien Center grant, by NIH grant DK065123, and by merit awards from the Department of Veterans Affairs. R. Zent is funded by NIH grants DK075594, DK65123, and DK083187 and by an American Heart Association established investigator award.

Address correspondence to: Roy Zent or Ambra Pozzi, Room C3210, Medical Center North, Vanderbilt University Medical Center, Nashville, Tennessee 37232-2372, USA. Phone: 615.322.4632; Fax: 615.322.4689;
E-mail: roy.zent@vanderbilt.edu (R.Zent); ambra.pozzi@vanderbilt.edu (A. Pozzi).

1. Campbell KN, Raij L, Mundel P. Role of angiotensin II in the development of nephropathy and podocytopathy of diabetes. Curr Diabetes Rev. 2011;7(1):3-7.

2 . Sachs $\mathrm{N}$, et al. Blood pressure influences end-stage renal disease of Cd151 knockout mice. J Clin Invest. 2012;122(1):348-358.

3. Baleato RM, Guthrie PL, Gubler MC, Ashman LK, Roselli S. Deletion of CD151 results in a straindependent glomerular disease due to severe alterations of the glomerular basement membrane. Am J Pathol. 2008;173(4):927-937

4. Pozzi A, Zent R. Extracellular matrix receptors in branched organs. Curr Opin Cell Biol. 2011;23(5):547-553.

5. Mathew S, Chen X, Pozzi A, Zent R. Integrins in renal development [published online ahead of print May 21, 2011]. Pediatr Nephrol. doi:10.1007/ s00467-011-1890-1.

6. Pozzi A, et al. Beta1 integrin expression by podocytes is required to maintain glomerular structural integrity. Dev Biol. 2008;316(2):288-301.
7. Kanasaki K, et al. Integrin beta1-mediated matrix assembly and signaling are critical for the normal development and function of the kidney glomerulus. Dev Biol. 2008;313(2):584-593.

8 . Sachs N, et al. Kidney failure in mice lacking the tetraspanin CD151. J Cell Biol. 2006;175(1):33-39.

9. Margadant C, Frijns E, Wilhelmsen K, Sonnenberg A. Regulation of hemidesmosome disassembly by growth factor receptors. Curr Opin Cell Biol. 2008;20(5):589-596.

10. Karamatic Crew V, et al. CD151, the first member of the tetraspanin (TM4) superfamily detected on erythrocytes, is essential for the correct assembly of human basement membranes in kidney and skin. Blood. 2004;104(8):2217-2223.

11. Wang HX, Li Q, Sharma C, Knoblich K, Hemler ME. Tetraspanin protein contributions to cancer. Biochem Soc Trans. 2011;39(2):547-552

12. Sawada S, Yoshimoto M, Odintsova E, Hotchin NA, Berditchevski F. The tetraspanin CD151 functions as a negative regulator in the adhesiondependent activation of Ras. J Biol Chem. 2003; 278(29):26323-26326.

13. Margadant C, Charafeddine RA, Sonnenberg A. Unique and redundant functions of integrins in the epidermis. FASEB J. 2010;24(11):4133-4152.

\title{
The flip-flop HuR: part of the problem or the solution in fighting cancer?
}

\author{
Jacqueline C. Shultz ${ }^{1}$ and Charles E. Chalfant ${ }^{1,2,3}$
}

\begin{abstract}
1Department of Biochemistry and Molecular Biology, Virginia Commonwealth University, Richmond, Virginia, USA ${ }^{2}$ Research and Development, Hunter Holmes McGuire Veterans Administration Medical Center, Richmond, Virginia, USA. ${ }^{3}$ The Massey Cancer Center, Virginia Commonwealth University, Richmond, Virginia, USA.
\end{abstract}

\begin{abstract}
Chronic inflammation has long been appreciated to play a critical role in tumor development and maintenance. Among the mechanisms involved in coordinating the initiation and resolution of inflammation are those responsible for modifying mRNA stability and/or translation. Several studies have linked the RNA-binding protein HuR, which increases mRNA stability, with malignant transformation. However, in this issue of the JCI, Yiakouvaki et al. compellingly demonstrate in mice that increased HuR activity in myeloid cells has a protective role in the onset of pathologic intestinal inflammation (i.e., colitis) and colitis-associated cancer (CAC). These observations highlight the need to understand the roles of $\mathrm{HuR}$ in distinct cell populations in vivo and suggest that enhancing $\mathrm{HuR}$ activity may be of clinical benefit in protecting against pathologic inflammation and cancer.
\end{abstract}

It has been clear for some time that chronic inflammation can promote and/or exacerbate tumor initiation, tumor promotion, and tumor progression (1). For example, clinical and epidemiologic studies have indicated that inflammation of the liver as a result of alcohol abuse or chronic infection with hepatitis $C$ virus is associated with hepatocellular carcinoma; inflam-

Conflict of interest: The authors have declared that no conflict of interest exists.

Citation for this article: J Clin Invest. 2012;

122(1):16-19. doi:10.1172/JCI61677. mation of the lung as a result of cigarette smoking, asbestos exposure, or silica exposure is associated with lung carcinoma; and inflammatory bowel diseases (IBDs) such as ulcerative colitis (UC) are associated with colon cancer. Defining the mechanisms responsible for coordinating the initiation and resolution of inflammation is therefore critical to understanding how inflammation-associated cancers arise and progress and to identifying potential therapeutic targets. Among the mechanisms responsible for coordinating the initiation and resolution of inflammation are those controlling the production and post-transcriptional regulation of mRNAs encoding inflammatory mediators. In this issue of the JCI (2), Yiakouvaki and colleagues provide a comprehensive study aimed at clarifying the role of one RNA-binding protein that controls mRNA stability and/or translation (HuR) in inflammation and inflammation-associated cancer.

\section{Post-transcriptional regulation of gene expression by HuR}

HuR is a member of the embryonic lethal, abnormal vision (ELAV) family of RNAbinding proteins that is encoded by the ELAV1 gene. It regulates gene expression via post-transcriptional mechanisms (3-5). Traditionally, HuR functions to modulate the stability and translation of target mRNAs that posses AU-rich elements (AREs) in their $3^{\prime}$-untranslated regions (UTRs). It has been previously demonstrated that myeloid HuR overexpression could induce translational silencing of AREcontaining transcripts, such as Tnfa, even though the mRNAs were stabilized (6). HuR is predominantly nuclear, but in response 
A $\mathrm{HuR}^{-}$myeloid cells

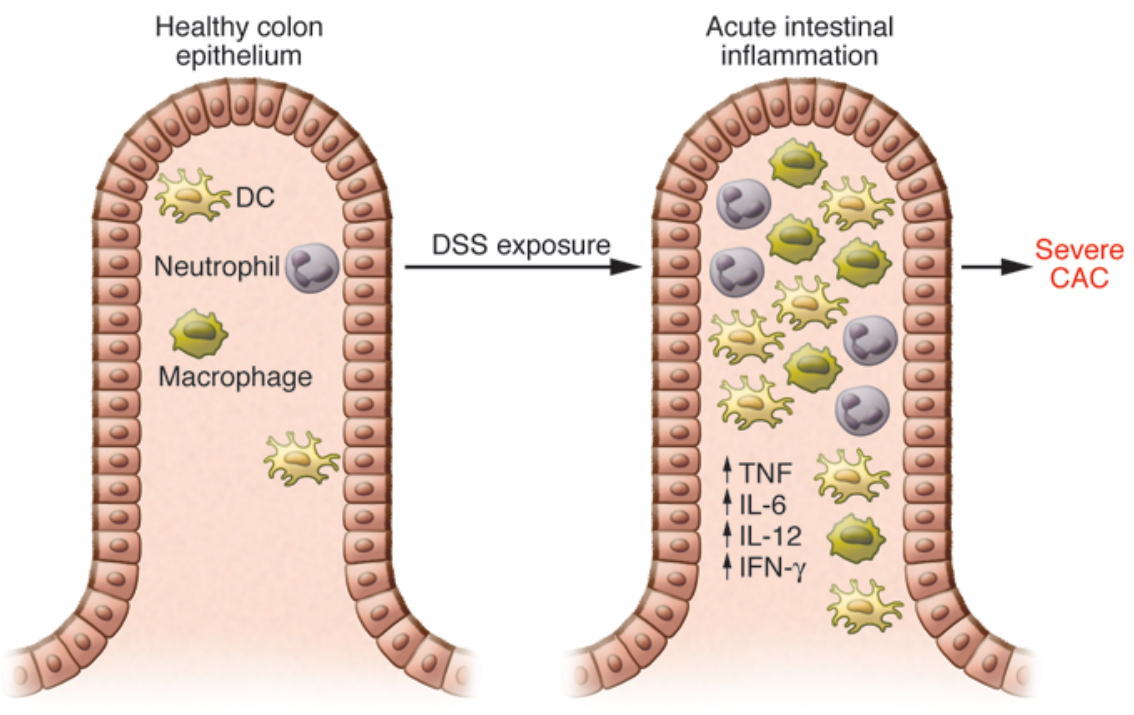

B HuR-overexpressing myeloid cells

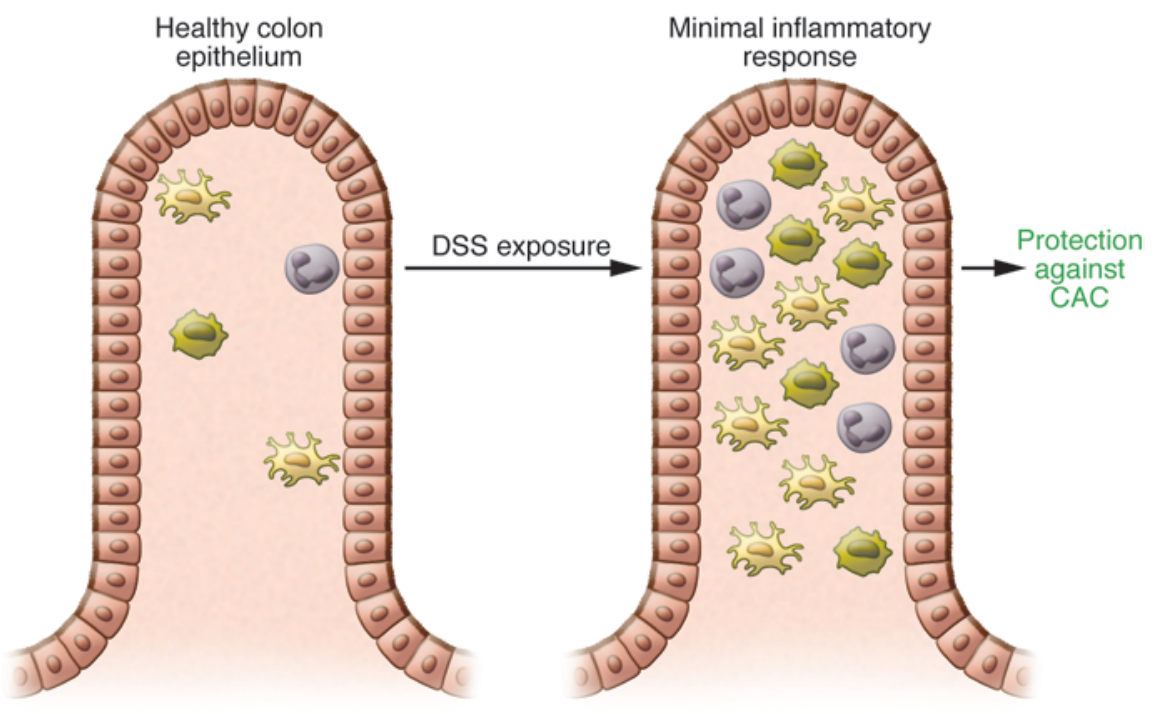

to various stimuli such as therapeutic agents (i.e., tamoxifen and prostaglandins), nutrient deprivation, and immune stimuli, HuR shuttles to the cytoplasm, where it acts to prolong target mRNA halflife and modulate target mRNA translation $(7,8)$. In vitro studies have demonstrated that $\mathrm{HuR}$ regulates the expression of genes involved in key cellular processes, including inflammation, tumorigenesis, the response to hypoxia, cell-cycle control, and the response to cell stress (3-5). HuR has also been demonstrated to promote cell survival by positively regulating the expression of antiapoptotic proteins and suppressing the expression of proapoptotic proteins (9-11). However, few studies have investigated the physiologic and pathologic functions of HuR in animal models, so the in vivo function of this RNA-binding protein is poorly understood. The work of Yiakouvaki and colleagues (2) provides some insight into this, specifically, HuR's function in myeloid cells, which are pivotal regulators of inflammation.

The role of HuR in the progression of colitis and colitis-associated cancer

While no mutations in the gene encoding HuR have been reported in cancer, studies have suggested a link between HuR and malignant transformation (12-14). Specifically, changes in HuR levels or localization in clinical samples from patients

\section{Figure 1}

Myeloid HuR alters the progression of colitis and CAC. Yiakouvaki and colleagues employed a mouse model of colon-specific inflammation induced by DSS to examine the effects of loss of HuR in myeloid cells and the overexpression of HuR in myeloid cells in regulating the progression of colitis (2). (A) Following exposure to DSS, mice devoid of HuR in the myeloid lineage (MKO mice) exhibited faster colitis onset and higher disease activity in comparison to control mice. Cultures and RNA extracts from the colons of MKO mice treated with DSS revealed increased expression of the proinflammatory cytokines TNF, IL-6, IL-12, and INF- $\gamma$ as compared with control mice treated with DSS. MKO mice were also sensitized to CAC and developed higher numbers of tumors as well as increased tumor size and grading as compared with control mice. (B) In contrast, the study by Yiakouvaki and colleagues indicates that mice overexpressing myeloid HuR exhibit delayed onset and severity of colitis and resistance to the development of CAC (2). with inflammatory disease or cancer have suggested that HuR functions in a proinflammatory and pro-tumorigenic manner $(14,15)$. Elevated levels of cytoplasmic HuR have been demonstrated to correlate with advanced malignancy and tumor progression for many cancers, including those of the colon (16-18). However, a number of recent studies have demonstrated that HuR might not always function in a protumorigenic manner. For example, overexpression of HuR was shown to impair tumor growth in an orthotopic mouse model of estrogen receptor-negative breast cancer (19). Likewise, in a clinical correlation study of the therapeutic efficacy of the first-line chemotherapeutic agent 
gemcitabine in individuals with pancreatic cancer, patients with low cytoplasmic levels of HuR exhibited a 7-fold increase in risk of mortality compared with patients with high cytoplasmic levels, suggesting that cytoplasmic HuR levels in pancreatic cancer modulate the therapeutic efficacy of gemcitabine (20). Therefore, the role of HuR in cancer remains unclear, as reports have suggested opposing roles in regard to tumor development and maintenance.

In this issue of the JCI (2), Yiakouvaki and colleagues have provided compelling evidence of a protective role for $\mathrm{HuR}$ in chronic inflammation and tumorigenesis. To examine the role of HuR in inflammatory responses, the authors induced autosomal inactivation of the Elavl1 locus in the mouse myeloid lineage to generate mice that they termed MKO mice. In a model of LPS-induced septic shock, MKO mice were found to be more susceptible to septic shock than controls; this was true for both mice of a susceptible genetic background and those of a resistant genetic background. Interestingly, the endotoxic response of MKO mice was inversely correlated with the enhanced presence of mRNAs encoding the proinflammatory cytokines TNF, IL-6, IL-1 $\beta$, and IL-12, confirming the induction of a heightened proinflammatory response. Macrophages cultured from MKO mice also revealed changes in the biosynthesis of key inflammatory mediators that could support inflammatory pathologies. Specifically, upregulation of Il6 mRNA and an accumulation of secreted IL- 6 protein, a key mediator of colitis-induced cancer, were observed, as was a similar response for IL-12, which drives inflammatory Th1 responses related to systemic and intestinal inflammation.

In an effort to examine the role of $\mathrm{HuR}$ in organ-specific inflammation, Yiakouvaki and colleagues employed a mouse model of colitis induced by DDS (2). Interestingly, MKO mice exhibited enhanced progression and maintenance of colitis (Figure 1). More striking were data obtained from experiments examining the role of myeloid HuR in a model of colitis-associated cancer (CAC) induced by dimethylhydrazine (DMH) and DSS. MKO mice exposed to DSS and DMH demonstrated increased tumor size and grading as compared with control groups. Importantly, Yiakouvaki and colleagues extended these results and examined whether HuR overexpression would block colitis and CAC (2). In contrast to control groups, DSS induced only a small inflammatory response in the colons of mice in which HuR was inducibly overexpressed. Most significant, however, DSS/DMH treatment of mice overexpressing $\mathrm{HuR}$ in the myeloid lineage resulted in the appearance of only a few small, low-grade adenomas and reduced tumor multiplicity, as compared with control groups. These experiments highlight the protective effects of myeloid HuR activity in regulating inflammatory tumorigenesis in the mouse colon.

\section{Therapeutic perspectives for treating pathologic inflammation and cancer}

As noted above, patients with UC are at increased risk for developing colorectal cancer, and chronic inflammation is the assumed culprit. Like other solid malignancies, colitis-associated tumors are infiltrated by a variety of immune cells, including neutrophils, mast cells, natural killer cells, dendritic cells, and tumorassociated macrophages (21). Previous studies have suggested that HuR has a role in the initiation and progression of colon cancer and the associated expression of immune modulators $(14,22,23)$, but now Yiakouvaki and colleagues have shed new light on the inflammatory component of the process. Specifically, they provide compelling evidence that increasing HuR functions in myeloid cells is an effective strategy to combat pathologic inflammation and cancer (2).

The notion of HuR as a therapeutic target is not new; the reports linking HuR to malignant transformation (12-14) had suggested that blocking the association of $\mathrm{HuR}$ with target ARE-containing mRNAs could decrease mRNA stability and thereby limit inflammation and inflammationassociated tumorigenesis. However, several recent reports $(19,20)$ as well as the work of Yiakouvaki and colleagues (2) have challenged this approach, suggesting that enhancing HuR activity, and not targeting specific ARE-containing mRNAs, could be of high clinical value against pathologic inflammation and cancer $(2,19)$.

\section{Concluding remarks}

Clearly, many questions remain. Among the most clinically relevant are: Is HuR overexpression protective in cancer types other than CACs and is the protection dependent on the presence of a strong inflammatory component? More investigation into the mechanism(s) by which
HuR acts to regulate inflammation and tumorigenesis as well as the potential interplay among other RNA-binding proteins would greatly add to the understanding of the in vivo function of HuR. Thus, future investigations based on the study by Yiakouvaki and colleagues may provide additional therapeutic targets to combat inflammation-associated cancers.

\section{Acknowledgments}

The production of this work was supported by grants from the Veteran's Administration (VA Merit Review I and a Research Career Scientist Award to C.E. Chalfant) and from the NIH (HL072925, CA117950, and CA154314 to C.E. Chalfant).

Address correspondence to: Charles E. Chalfant, Department of Biochemistry and Molecular Biology, Room 2-016, Sanger Hall, Virginia Commonwealth University - School of Medicine, 1101 East Marshall Street, P.O. Box 980614, Richmond, Virginia 23298-0614, USA. Phone: 804.828.9526; Fax: 804.828.1473; E-mail: cechalfant@vcu.edu.

1. Keibel A, Singh V, Sharma MC. Inflammation, microenvironment, and the immune system in cancer progression. Curr Pharm Des. 2009; 15(17):1949-1955.

2. Yiakouvaki A, Dimitriou M, Karakasiliotis I, Eftychi C, Theocharis S, Kontoyiannis DL. Myeloid cell expression of the RNA-binding protein HuR protects mice from pathologic inflammation and colorectal carcinogenesis. J Clin Invest. 2012; 122(1):48-61

3. Abdelmohsen K, Lal A, Kim HH, Gorospe M. Posttranscriptional orchestration of an anti-apoptotic program by HuR. Cell Cycle. 2007;6(11):1288-1292.

4. Brennan CM, Steitz JA. HuR and mRNA stability. Cell Mol Life Sci. 2001;58(2):266-277.

5. Gorospe M. HuR in the mammalian genotoxic response: post-transcriptional multitasking. Cell Cycle. 2003;2(5):412-414.

6. Katsanou V, et al. HuR as a negative posttranscriptional modulator in inflammation. Mol Cell. 2005; 19(6):777-789.

7. Hostetter C, et al. Cytoplasmic accumulation of the RNA binding protein HuR is central to tamoxifen resistance in estrogen receptor positive breast cancer cells. Cancer Biol Ther. 2008;7(9):1496-1506.

8. Hinman MN, Lou H. Diverse molecular functions of Hu proteins. Cell Mol Life Sci. 2008; 65(20):3168-3181.

9. Mazan-Mamczarz K, et al. RNA-binding protein HuR enhances p53 translation in response to ultraviolet light irradiation. Proc Natl Acad Sci U S A. 2003; 100(14):8354-8359.

10. Zou T, et al. Polyamine depletion increases cytoplasmic levels of RNA-binding protein HuR leading to stabilization of nucleophosmin and p53 mRNAs. J Biol Chem. 2006;281(28):19387-19394.

11. Tong X, Pelling JC. Enhancement of p53 expression in keratinocytes by the bioflavonoid apigenin is associated with RNA-binding protein HuR. Mol Carcinog. 2009;48(2):118-129.

12. Lopez de Silanes I, Fan J, Galban CJ, Spencer RG, Becker KG, Gorospe M. Global analysis of HuR- 
regulated gene expression in colon cancer systems of reducing complexity. Gene Expr. 2004;12(1):49-59.

13. Lopez de Silanes I, et al. Role of the RNA-binding protein $\mathrm{HuR}$ in colon carcinogenesis. Oncogene. 2003; 22(46):7146-7154

14. Lopez de Silanes I, Lal A, Gorospe M. HuR: posttranscriptional paths to malignancy. RNA Biol. 2005; 2(1):11-13

15. Rhee WJ, Ni CW, Zheng Z, Chang K, Jo H, Bao G. HuR regulates the expression of stress-sensitive genes and mediates inflammatory response in human umbilical vein endothelial cells. Proc Natl Acad Sci U S A. 2010;107(15):6858-6863

16. Yoo PS, et al. Tissue microarray analysis of 560 patients with colorectal adenocarcinoma: high expression of HuR predicts poor survival. Ann Surg
Oncol. 2009;16(1):200-207.

17. Denkert C, et al. Expression of the ELAV-like protein $\mathrm{HuR}$ is associated with higher tumor grade and increased cyclooxygenase- 2 expression in human breast carcinoma. Clin Cancer Res. 2004; 10(16):5580-5586.

18. Denkert C, et al. Overexpression of the embryoniclethal abnormal vision-like protein HuR in ovarian carcinoma is a prognostic factor and is associated with increased cyclooxygenase 2 expression. Cancer Res. 2004;64(1):189-195.

19. Gubin MM, et al. Overexpression of the RNA binding protein HuR impairs tumor growth in triple negative breast cancer associated with deficient angiogenesis. Cell Cycle. 2010;9(16):3337-3346.

20. Costantino CL, et al. The role of HuR in gemcitabi- ne efficacy in pancreatic cancer: HuR Up-regulates the expression of the gemcitabine metabolizing enzyme deoxycytidine kinase. Cancer Res. 2009; 69(11):4567-4572.

21. Atreya I, Neurath MF. Immune cells in colorectal cancer: prognostic relevance and therapeutic strategies. Expert Rev Anticancer Ther. 2008;8(4):561-572.

22. Lim SJ, Lee SH, Joo SH, Song JY, Choi SI. Cytoplasmic expression of HuR is related to cyclooxygenase-2 expression in colon cancer. Cancer Res Treat. 2009; 41(2):87-92.

23. Young LE, Sanduja S, Bemis-Standoli K, Pena EA, Price RL, Dixon DA. The mRNA binding proteins HuR and tristetraprolin regulate cyclooxygenase 2 expression during colon carcinogenesis. Gastroenterology. 2009;136(5):1669-1679.

\title{
Epoxyeicosatrienoic acids: a double-edged sword in cardiovascular diseases and cancer
}

\author{
Dingzhi Wang1 and Raymond N. DuBois ${ }^{1,2}$
}

${ }^{1}$ Department of Cancer Biology and 2Department of Gastrointestinal Medical Oncology, The University of Texas MD Anderson Cancer Center, Houston, Texas, USA.

\begin{abstract}
Epoxyeicosatrienoic acids (EETs) generated from arachidonic acid by cytochrome P450 (CYP) epoxygenases have beneficial effects in certain cardiovascular and kidney diseases. Hence, great efforts have been made to develop drugs targeting the EET pathway. Some of these agents are currently under evaluation in clinical trials for treatment of hypertension and diabetes. In this issue of the JCI, Panigrahy et al. evaluate in a systematic way the role of CYP epoxygenases and the metabolites they generate in cancer progression. Their findings, along with previous studies, raise concerns about using these drugs in humans.
\end{abstract}

High dietary fat intake is reported to be associated with several human diseases, including diabetes and heart disease. Moreover, epidemiologic and experimental observations support the hypothesis that high dietary fat intake is also a risk factor for cancers. However, the mechanisms underlying the link between high dietary fat intake and cancer progression are poorly understood. One factor thought to be involved is arachidonic acid (AA), a major component of animal fats that is primarily found in red meats, egg yolks, and organ meats. The bioactive lipids derived from AA play critical roles in cancer progression (1).

\section{Eicosanoid synthesis pathways}

AA is a polyunsaturated omega- 6 fatty acid that constitutes the phospholipid domain of most cell membranes. AA is liberated by cytoplasmic phospholipase $\mathrm{A}_{2}$ (cPLA2)

Conflict of interest: The authors have declared that no conflict of interest exists.

Citation for this article: J Clin Invest. 2012; 122(1):19-22. doi:10.1172/JCI61453.
(Figure 1). Free AA can be metabolized to eicosanoids through three major pathways: the prostaglandin-endoperoxide synthase/ cyclooxygenase (PTGS/COX) pathway, the lipoxygenase (LOX) pathway, and the cytochrome P450 (CYP) pathway. Prostanoids are the eicosanoids generated by the PTGS/COX pathway, while the LOX pathway generates leukotrienes and hydroxyeicosatetraenoic acids (HETEs). The CYP enzymes that convert AA into eicosanoids include CYP epoxygenase and CYP $\omega$-hydroxylase enzymes. CYP epoxygenases, such as members of the CYP2C and CYP2J families, metabolize AA to four biologically active epoxyeicosatrienoic acids (EETs) (5,6-EET, 8,9-EET, 11,12EET, and 14,15-EET). CYP $\omega$-hydroxylases, such as members of the CYP4A and CYP4F families, convert AA to HETEs. Among the members of the CYP2C and CYP2J families of CYP enzymes, CYP2J2, CYP2C8, and CYP2C9 are the predominant epoxygenase isoforms that convert AA into EETs. All EETs are then further metabolized to less active dihydroxyeicosatrienoic acids (DHETs) by soluble epoxide hydrolase (sEH).

\section{EET signaling in cardiovascular and kidney diseases}

EETs are produced primarily by endothelial cells, although they are also produced by other cell types, such as astrocytes and cardiomyocytes. Because EETs induce vasodilation and exert antiinflammatory effects in blood vessels in an autocrine manner (2), they can lower blood pressure, protect the myocardium and brain from ischemia, attenuate hypertension-induced renal damage, and reduce cigarette smokeinduced lung inflammation (3). Increasing evidence reveals that EETs govern these various biological functions by inducing endothelial cell proliferation, survival, and tube formation and stimulating renal epithelial cell proliferation and survival through multiple signaling pathways (4). Hence, great efforts have been made to develop drugs targeting these pathways. For example, 11,12-EET has been shown to improve coronary artery endothelial function when it is added to transplant preservation solutions (5). Moreover, an sEH inhibitor (AR9281) is currently under evaluation in phase II clinical trials as a treatment for patients with hypertension and type 2 diabetes on the basis of evidence that sEH inhibitors have beneficial effects in animal models of hypertension and cardiovascular diseases $(3,6)$. However, emerging evidence shows that EETs can promote cancer progression by directly promoting cancer cell proliferation, survival, migration, and invasion. In this issue of the JCI, 